\title{
Analysis of the efficacy of intensity-modulated radiotherapy and two-dimensional conventional radiotherapy in nasopharyngeal carcinoma with involvement of the cervical spine
}

\author{
HAO JIANG ${ }^{1}$, GENGMING WANG ${ }^{1}$, HONGWEI SONG $^{2}$, HONGBO XU $^{1}$, YAJUN ZHANG ${ }^{1}$, \\ YUFU ZHOU ${ }^{1}$, HANFEI CAI ${ }^{1}$ and SHIMIAO DUAN ${ }^{2}$ \\ Departments of ${ }^{1}$ Radiation Oncology and ${ }^{2}$ Radiology, The First Affiliated Hospital of Bengbu Medical College, \\ Bengbu, Anhui 233004, P.R. China
}

Received September 13, 2014; Accepted July 7, 2015

DOI: $10.3892 / \mathrm{ol} .2015 .3668$

\begin{abstract}
The aim of the present study was to retrospectively analyze the clinical efficacy and side-effects of two-dimensional conventional radiotherapy (2D-CRT) and intensity-modulated radiotherapy (IMRT) in 53 NPC patients with cervical spine involvement, without distant metastases. In total, 53 patients were enrolled in the present study, with 24 being treated with IMRT and 29 being treated with 2D-CRT. All 53 patients received platinum-based concurrent chemotherapy and 4-6 cycles of adjuvant chemotherapy subsequent to radiation. The patients were clinically staged according to the seventh edition of the UICC and AJCC staging systems. Overall survival (OS), local progression-free survival (LPFS) and distant metastasis-free survival (DMFS) rates were calculated. The 3- and 5-year OS rates were $87.7 \%$ and $45.5 \%$ in the IMRT-treated group and $65.5 \%$ and $9.1 \%$ in the 2D-CRT-treated group $(\mathrm{P}=0.01)$. The 3- and 5-year LPES rates were $87.4 \%$ and $69.9 \%$ in the IMRT-treated group compared with $49.4 \%$ and $9.4 \%$ in the 2D-CRT-treated group,
\end{abstract}

Correspondence to: Professor Hao Jiang, Department of Radiation Oncology, The First Affiliated Hospital of Bengbu Medical College, 287 Changhuai Road, Bengbu, Anhui 233004, P.R. China

E-mail: haojiangcn@126.com

Abbreviations: CR, complete response; CTV1, clinical target volume 1; CTV2, clinical target volume 2; CTVnd, clinical target volume of the visible lymph nodes; DDP, cisplatin; DMFS, distant metastasis-free survival; GTVnx, gross tumor volumes of nasopharyngeal tumor; GTVnd, gross tumor volumes of the cervical lymph nodes; IMRT, intensity-modulated radiotherapy; LPFS, local progression-free survival; MRI, magnetic resonance imaging; NPC, nasopharyngeal carcinoma; OAR, organ at risk; OS, overall survival; 2D-CRT, two-dimensional conventional radiotherapy; 5-Fu, fluorouracil

Key words: nasopharyngeal carcinoma, cervical spine involvement, intensity-modulated radiotherapy, two-dimensional conventional radiotherapy, prognosis respectively $(\mathrm{P}=0.00)$. The 3 - and 5-year DMFS rates were 94.4 and $40.8 \%$ in the IMRT-treated group and 79.8 and $30.4 \%$ in the 2D-CRT-treated group $(\mathrm{P}=0.13)$. $\mathrm{N}$ stage $(\mathrm{P}=0.00)$ and radiotherapy methods $(\mathrm{P}=0.01)$ were relevant to the $\mathrm{OS}$ and LPFS rates, it also revealed a significant difference when the DMFS rates were analyzed in $\mathrm{N}$ stage. The incidence of dry mouth in the IMRT group was significantly lower $(\mathrm{P}=0.01)$, but there was no statistically significant difference in acute oropharyngeal mucositis or myelosuppression. IMRT had significant advantages in local control and OS compared with conventional 2D-CRT, but IMRT failed to reduce the incidence of distant metastasis.

\section{Introduction}

Nasopharyngeal carcinoma (NPC) is a common malignancy in mainland China, with an age-standardized incidence of 13.00-30.94 cases per 100,000 person-years and a five-year survival rate of $\sim 70 \%$ (1). Cervical spine involvement is rare in patients with NPC, and concurrent chemoradiotherapy is the primary treatment strategy for NPC patients with or without cervical spine involvement (2-4). The clinical efficacy of treatment for NPC is significantly decreased in patients with locally advanced disease. In particular, the clinical efficacy is decreased in patients with NPC advancing toward the cervical spine, which occurs in 4-6\% of NPC patients (5-7). According to the domestic 1992 Fuzhou staging methods, cervical spine involvement results in NPC being classified as stage T4. However, this was no longer considered as a clinical indicator of $\mathrm{T}$ staging in the UICC/AJCC NPC staging criteria (8).

In NPC patients with cervical spine involvement, the proximity between the tumor and the cervical spinal cord makes it challenging to balance tumor therapy and spinal cord tolerance using two dimensional-conventional radiotherapy (2D-CRT). The doses of radiation administered to the targeted tumor must be reduced to prevent the occurrence of severe radiation myelitis. The radiation dose to cervical vertebra involved by tumors may be significantly increased by the conformal intensity-modulated radiotherapy (IMRT) technique. In theory, IMRT has unparalleled advantages in increasing the 
local radiotherapy dose compared to 2D-CRT to treat NPC with cervical spine involvement. IMRT was employed to treat NPC in 2006. Since then, 53 NPC patients with cervical spine involvement without distant metastases were treated with 2D-CRT or IMRT, and the retrospective analyses of these patients are described in the present study.

\section{Patients and methods}

Patients. Between January 2006 and December 2012, 53 NPC patients with cervical spine involvement without distant metastasis were enrolled in the present study, accounting for 5.75\% of the total 921 patients that underwent treatment for NPC at The First Affiliated Hospital of Bengbu Medical College (Bengbu, Anhui, China). The patients consisted of 37 males and 16 females aged 12-72 years, with a median age of 45 years. In total, 24 patients underwent IMRT and 29 patients underwent 2D-CRT, based on individual preference. All patients were treated with two cycles of concurrent chemotherapy followed by four to six cycles of adjuvant chemotherapy. The patients treated prior to 2009 were re-staged by magnetic resonance imaging (MRI) and clinical data according to the UICC/AJCC staging criteria (8). The distribution of clinical data and comparability test results of the two groups are reported in Table I. There were no significant differences in the distribution of clinical data between the two groups, indicating that they were comparable. The present study was conducted in accordance with the Declaration of Helsinki (9) and with approval from the Ethics Committee of the First Affiliated Hospital of Bengbu Medical College.

Definition of cervical spine involvement. All patients underwent plain and enhanced magnetic resonance imaging (MRI) examination using conventional spin-echo (SE) sequences prior to radiotherapy. The cross-sectional, sagittal and coronal planes were used in scanning. Two personnel from the MRI and Radiotherapy Departments of The First Affiliated Hospital of Bengbu Medical College cooperated to determine whether the patients demonstrated cervical spine involvement. The criteria for the determination of cervical vertebrae involvement were as follows. The low signal of normal structure disappeared in cortical bone scan and was replaced by low signal on T1-weighted imaging (T1WI) and slightly higher signal of tumor tissue on T2-weighted imaging (T2WI), which was significantly intensified on T1WI combined with fat suppression (FS) subsequent to FS enhancement. Additionally, the identification of low signal of cancellous bone structure on T1WI and disappearance of a slightly higher signal on T2WI, which was replaced by the low signal for soft tissue on T1WI and slightly higher signal on T2WI (patchy heterogeneous signal) that were also significantly intensified on T1WI combined with FS subsequent to FS enhancement was required $(6,7)$.

Radiotherapy. Radiotherapy was performed using an Elekta Precise (Elekta, Stockholm, Sweden) or a Siemens Artistes ART linear accelerator (Siemens, Munich, Germany). The radiotherapy protocol was designed by Philips pinnacle 7.0-8.2 treatment planning system (TPS; Philips, Best, Netherlands).
The 2D-CRT protocol involved the use of an X-ray beam from a 6-MV high-energy linear accelerator in order to treat primary NPC. X-rays from a 6-MV high-energy accelerator and an 8-15 MeV electron beam were used in combination to treat lymph drainage areas, and the specific ratio was determined based on the tumor size. The radiation dose of conventional radiotherapy was 2.0 Gy per fraction and 1 fraction daily, which was administered 5 days per week. The faciocervical joint field in the pharynx nasalis was irradiated with doses of 36-40 Gy. In the back-reduced field, the spinal cord was avoided, and the radiation field boundary was the posterior one-third of cervical vertebrae, with additional doses of 68-74 Gy (median, $70 \mathrm{~Gy}$ ). The total doses for cervical lymph node metastasis were 66-70 Gy. Those patients with positive cervical lymph node involvement underwent whole-neck irradiation, while those with no involved cervical lymph nodes were administered with semi-neck prophylactic irradiation at doses of 50-54 Gy.

IMRT was used for the treatment of primary NPC and the neck area. The target volumes were delineated according to the treatment protocol of The First Affiliated Hospital of Bengbu Medical College, in agreement with the International Commission on Radiation Units and Measurements Reports 50 and 62 (10). The target areas defined consisted of the gross tumor volumes of the nasopharyngeal tumor (GTVnx), gross tumor volumes of the cervical lymph nodes (GTVnd), clinical target volume 1 of high-risk regions (CTV1), clinical target volume 2 of low-risk regions (CTV2), clinical target volume of the neck lymph nodes (CTVnd), planning target volume and organ at risk (OAR). GTV was delineated based on fused computed tomography (CT) and MRI images, since the cervical vertebrae were affected by the tumor. CTV1 and CTV2 were broadened by $3-5 \mathrm{~mm}$ and 5-8 $\mathrm{mm}$, respectively, from the GTV and toward the spinal cord. The weight of the spinal cord was increased compared with the GTV when planned with TPS, and the spinal cord exposure dose was defined as $>45 \mathrm{~Gy}$, with a volume $\leq 5 \%$. Each spinal cord plane was assessed to ensure that a surrounding cross-sectional area with $>45$ Gy was $\leq 10 \%$ of the sectional area of the spinal cord at this level. The brainstem exposure dose was defined as a volume of $>54$ Gy being $\leq 5 \%$, and each brainstem plane was assessed to ensure that a surrounding cross-sectional area with $>45$ Gy was $\leq 10 \%$ of the sectional area of the brainstem on this level. The defined target doses were as follows: GTVnx, 72-74 Gy, 33-35 fractions, over 6-7 weeks; CTV1, 60-64 Gy; CTV2, 54-56 Gy; GTVnd, 66-72 Gy; and CTVnd, 50-56 Gy. For those patients with no involved lymph nodes, only the lymph nodes above the cricoid cartilage were defined as the CTVnd, while the whole neck area was defined as the CTVnd in those patients with positive local lymph node metastasis.

Concurrent chemotherapy. Concurrent chemotherapy consisted of a cisplatin-based chemotherapy protocol comprising cisplatin (DDP) plus fluorouracil (5-Fu). The DDP dose was $20 \mathrm{mg} / \mathrm{m}^{2}$, administered on days $1-3$, and the 5 -Fu dose was $600 \mathrm{mg} / \mathrm{m}^{2}$, administered on days $1-5$. The two agents were administered at the beginning of radiation therapy and then at 5 weeks subsequent to the administration of radiation therapy. If patients experienced any severe 
Table I. Comparison of the clinical data of 53 patients with nasopharyngeal carcinoma treated using IMRT or 2D-CRT.

\begin{tabular}{|c|c|c|c|c|}
\hline Clinical data & IMRT & 2D-CRT & $\chi^{2}$ value & P-value \\
\hline Total & 24 & 29 & & \\
\hline \multicolumn{5}{|l|}{ Gender } \\
\hline Male & 19 & 18 & 1.79 & 0.18 \\
\hline Female & 5 & 11 & & \\
\hline \multicolumn{5}{|l|}{ Age } \\
\hline$\leq 45$ & 14 & 14 & 0.52 & 0.47 \\
\hline$>45$ & 10 & 15 & & \\
\hline \multicolumn{5}{|l|}{ Treatment year } \\
\hline Prior to 2009 & 9 & 14 & 1.05 & 0.31 \\
\hline After 2009 & 15 & 15 & & \\
\hline \multicolumn{5}{|l|}{ T stage } \\
\hline $\mathrm{T} 3$ & 4 & 6 & 0.14 & 0.71 \\
\hline $\mathrm{T} 4$ & 20 & 23 & & \\
\hline \multicolumn{5}{|l|}{$\mathrm{N}$ stage } \\
\hline N0 & 4 & 5 & 0.03 & 0.87 \\
\hline N1 & 7 & 10 & & \\
\hline N2 & 8 & 8 & & \\
\hline N3 & 5 & 6 & & \\
\hline \multicolumn{5}{|l|}{ Clinical stage } \\
\hline III & 4 & 5 & 0.00 & 0.96 \\
\hline IVa & 20 & 24 & & \\
\hline \multicolumn{5}{|l|}{ Pathological type } \\
\hline $\begin{array}{l}\text { Undifferentiated } \\
\text { non-keratinizing } \\
\text { carcinoma }\end{array}$ & 22 & 26 & 0.06 & 0.81 \\
\hline $\begin{array}{l}\text { Differentiated } \\
\text { non-keratinizing } \\
\text { carcinoma }\end{array}$ & 2 & 2 & & \\
\hline Keratinizing carcinoma & 0 & 1 & & \\
\hline
\end{tabular}

IMRT, intensity-modulated radiotherapy; 2D-CRT, two-dimensional conventional radiotherapy.

radiation reactions, such as myelosuppression or oral mucositis of level 3 or higher, the concurrent chemotherapy was be terminated based on clinical assessment and the preference of the patient. The second concurrent chemotherapy regimen was not implemented in 3 patients, consisting of 2 patients in the 2D-CRT group and 1 patient in the IMRT group, respectively.

Adjuvant chemotherapy. All patients underwent six cycles of adjuvant chemotherapy subsequent to radiotherapy. The DDP plus 5-Fu chemotherapy regimen was used. The DDP dose was $25-30 \mathrm{mg} / \mathrm{m}^{2}$, administered on days $1-4$, and the 5 -Fu dose was $600 \mathrm{mg} / \mathrm{m}^{2}$, administered on days $1-5$. In total, 4 patients, with 2 patients from each group, were treated with DDP plus docetaxel, in which the DDP dose of $25-30 \mathrm{mg} / \mathrm{m}^{2}$ was administered on days 1-4 and the docetaxel dose of $80 \mathrm{mg} / \mathrm{m}^{2}$ was administered on day 1 .
Tumor response assessment and criteria. The response of the tumor to treatment was assessed following the end of all treatments. The local control and systemic metastasis status were evaluated by enhanced MRI examination and other systemic examinations, including chest CT and liver ultrasonography. Efficacy of treatment in the nasopharynx and neck was evaluated by enhanced MRI. Complete response (CR) was considered to have occurred if the tumor had regressed and the primary mass image disappeared completely on T2WI+FS. Partial response was considered to have occurred if tumor remnants were present, but the tumor volume had reduced $>50 \%$. Stable disease was diagnosed if the volume of the tumor had reduced $<50 \%$. Tumor progression was considered to indicate progressive disease.

Follow-up. Follow-up was performed mainly by subsequent visits and supplemented by telephone follow-up. The patients were followed up every 3 to 4 months for 3 years subsequent to treatment, and then every 6 months. This was decreased to at least once a year subsequent to 5 years. The last date of this follow-up was May 31, 2013, and there was no study attrition.

Statistical analysis. The date of radiotherapy initiation was the beginning time for the statistical survival analysis. Tumor progression, local tumor progression, distant metastasis, and mortality were the statistical endpoints of tumor progression-free survival, local progression-free survival, distant metastasis-free survival and overall survival, respectively. These values were used to calculate the 1-, 3- and 5-year survival rates of patients. Statistical analysis of survival was performed using the Kaplan-Meier and log-rank analyses, while factors associated with survival were analyzed using the $\chi^{2}$ test and Cox regression. Calculations were performed using SPSS 19.0 (IBM, Armonk, NY, USA) and the specific statistical methods are explained in the table legends. $\mathrm{P}<0.05$ was considered to indicate a statistically significant difference.

\section{Results}

$C R$ and side-effects of treatment. At the end of the treatment, the final CR rate of local cancer treatment was $66.7 \%$ $(16 / 24)$ in the IMRT group and $24.1 \%$ in the 2D-CRT group (7/29), and these values were significantly different $\left(\chi^{2}=9.67\right.$; $\mathrm{P}=0.00)$. With regard to side-effects, there was no statistical difference between the two groups for radiation myelitis, oropharyngeal mucositis or myelosuppression, but there was a significant difference in the prevalence of dry mouth between the two groups (Table II).

Analysis of the cause of tumor progression. In the present study, the follow-up time was 6-83 months, with a median follow-up time of 39 months. Out of the 53 patients, tumor progression occurred in 32 patients during the follow-up period, with 2 patients demonstrating local progression and distant metastasis. In total, 21 patients demonstrated local treatment failure, with 3 and 18 cases occurring in the IMRT and 2D-CRT groups, respectively, and the incidence in IMRT group was significantly decreased compared with the incidence in the 2D-CRT group $\left(\chi^{2}=13.49 ; \mathrm{P}=0.00\right)$. There were 13 patients that developed distant metastasis, consisting of 
Table II. Response to cancer treatment and side-effects following therapy in 53 patients with nasopharyngeal carcinoma.

\begin{tabular}{|c|c|c|c|c|}
\hline Clinical data & IMRT & 2D-CRT & $\chi^{2}$ value & P-value \\
\hline Total & 24 & 29 & & \\
\hline \multicolumn{5}{|l|}{$\begin{array}{l}\text { Cancer treatment } \\
\text { response }\end{array}$} \\
\hline Tumor regression & 16 & 7 & 9.67 & 0.00 \\
\hline Tumor residue & 8 & 22 & & \\
\hline \multicolumn{5}{|l|}{$\begin{array}{l}\text { Radiation myelitis } \\
\text { grade }\end{array}$} \\
\hline 0 & 22 & 28 & 0.58 & 0.45 \\
\hline 1 & 2 & 1 & & \\
\hline \multicolumn{5}{|l|}{ Dry mouth } \\
\hline Degree 0-1 & 17 & 10 & 7.11 & 0.01 \\
\hline Degree 2 & 6 & 14 & & \\
\hline Degree 3 & 1 & 5 & & \\
\hline \multicolumn{5}{|l|}{$\begin{array}{l}\text { Oral and pharyngeal } \\
\text { mucositis grade }\end{array}$} \\
\hline $0-1$ & 4 & 3 & 0.13 & 0.72 \\
\hline 2 & 8 & 9 & & \\
\hline$\geq 3$ & 12 & 17 & & \\
\hline \multicolumn{5}{|l|}{$\begin{array}{l}\text { Myelosuppression } \\
\text { grade }\end{array}$} \\
\hline 0 & 5 & 7 & 0.14 & 0.71 \\
\hline 1 & 11 & 14 & & \\
\hline 2 & 6 & 5 & & \\
\hline 3 & 2 & 3 & & \\
\hline
\end{tabular}

IMRT, intensity-modulated radiotherapy; 2D-CRT, two-dimensional conventional radiotherapy.

5 and 8 patients in the IMRT and 2D-CRT groups, respectively, but this was not significantly different between the two groups $\left(\chi^{2}=0.32 ; \mathrm{P}=0.57\right)$.

Survival analysis. The data revealed that the 3- and 5-year OS rates in the IMRT group were 87.7 and $45.5 \%$, respectively. In the 2D-CRT group, the 3- and 5-year OS rates were 65.5 and $9.1 \%$, respectively. The difference between the two groups was significant $\left(\chi^{2}=6.89 ; \mathrm{P}=0.01\right.$; Fig. 1$)$. However, the 3 - and 5-year LPFS rates were 87.4 and $69.9 \%$, respectively, in the IMRT group, and 49.4 and $9.4 \%$, respectively, in the 2D-CRT group. There was a significant intergroup difference $\left(\chi^{2}=13.26\right.$; $\mathrm{P}=0.00)$. The 3- and 5-year DMFS rates were 94.4 and $40.8 \%$ in the IMRT group and 79.8 and $30.4 \%$ in the 2D-CRT group, respectively ( $\chi^{2}=2.29 ; \mathrm{P}=0.13$; Figs. 2 and 3$)$.

Univariate analysis of patient gender, patient age, treatment time, case types, $\mathrm{T}$ stage, $\mathrm{N}$ stage, tumor-node-metastasis (TNM) stage and radiotherapy methods revealed that the $\mathrm{N}$ stage and radiotherapy method were each significantly associated with the OS and PFS rates of patients with NPC and cervical spine involvement (Table III). Multivariate Cox regression analysis also revealed that the $\mathrm{N}$ stage and radiotherapy method used were factors associated with the OS and

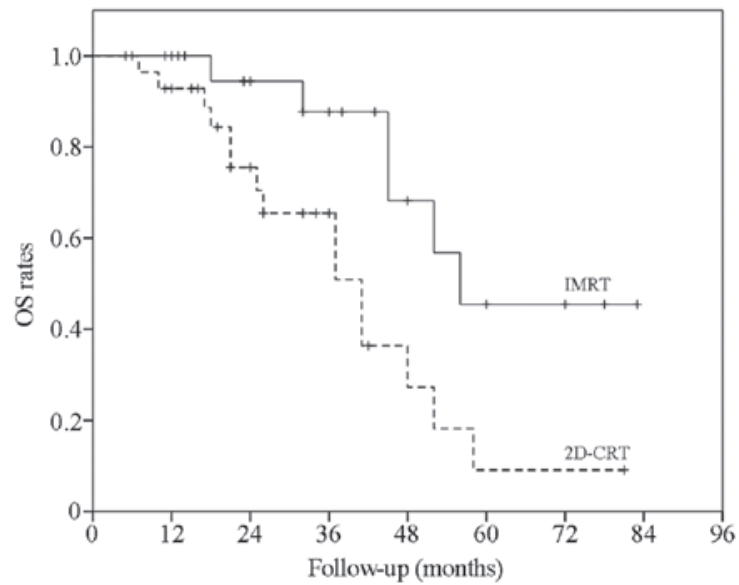

Figure 1. OS curves in the IMRT and 2D-CRT groups. The OS rates were assessed using the Kaplan-Meier and log-rank analyses. The OS rates in the two groups were signifcantly different $\left[\chi^{2}=6.89 ; \mathrm{P}=0.01\right.$; IMRT 95\% confidential interval (CI), 49.87-74.02 months; 2D-CRT 95\% CI, 30.67-45.82 months]. IMRT, intensity-modulated radiotherapy; 2D-CRT, two-dimensional conventional radiotherapy; OS, overall survival.

LPFS rates $(\mathrm{P}<0.05)$. Only the DMFS rate analyzed in terms of the $\mathrm{N}$ stage demonstrated a significant difference, but other factors, such as the radiotherapy method used, demonstrated no difference $(\mathrm{P}>0.05$; Table IV).

\section{Discussion}

Cervical spine involvement is unusual in NPC, with an incidence of $\sim 5 \%$, which is considerably decreased compared with the incidence of basicranial involvement (30-50\%). Cervical spine involvement is mainly clinically diagnosed through CT, MRI, positron emission tomography-CT and physical examination (5-7). Poon et al (6) identified in 107 patients that the imaging detection rates of NPC cervical spine involvement by CT and MRI were $0.93 \%$ (1/107) and 3.74\% (4/107), respectively. Zhang et al (7) assessed 250 patients and reported that the rates of NPC cervical spine involvement that were detected using CT and MRI were 0.80\% (2/250) and 6.4\% (16/250), respectively. The data in the two aforementioned studies revealed that enhanced MRI was more effective in detecting NPC cervical spine involvement compared with CT. The incidence of cervical spine involvement determined by MRI in the present study was $5.75 \%$ (53/921), which is similar to that reported in the literature.

The presence of cervical spine involvement results in tumors being defined as stage $\mathrm{T} 4$, according to the Chinese 1992 Fuzhou staging criteria (11), but this was not specified in the 2009 UICC NPC staging criteria. Out of the 53 patients in the present study, 10 patients were defined as possessing stage T3 disease, as they demonstrated simple cervical spine involvement, and the other 43 patients were defined as possessing stage T4 disease, as they demonstrated cervical spine involvement with concomitant cranial nerve injury, pterygoid muscle invasion or intracranial invasion. The clinical features of the patients are listed in Table I. This staging also affected the final TNM staging. Out of the 10 patients that were defined as possessing stage T3 disease according to the UICC/AJCC 2009 staging criteria (8), 1 patient remained classified as 
Table III. Univariate analysis of the clinical data of 53 patients with nasopharyngeal carcinoma.

\begin{tabular}{|c|c|c|c|c|c|c|c|}
\hline Clinical data & $\begin{array}{c}\text { Cases, } \\
\mathrm{n}\end{array}$ & $\begin{array}{l}\text { 5-year overall } \\
\text { survival rate, \% }\end{array}$ & $\chi^{2}$ value & P-value & $\begin{array}{l}\text { 5-year progression } \\
\text { free survival rate, } \%\end{array}$ & $\chi^{2}$ value & P-value \\
\hline \multicolumn{8}{|l|}{ Gender } \\
\hline Male & 37 & 26.6 & \multirow[t]{2}{*}{0.00} & \multirow[t]{2}{*}{0.99} & 32.7 & \multirow[t]{2}{*}{0.00} & \multirow[t]{2}{*}{0.99} \\
\hline Female & 16 & 21.0 & & & 29.0 & & \\
\hline \multicolumn{8}{|l|}{ Age, years } \\
\hline$\leq 45$ & 28 & 20.2 & \multirow[t]{2}{*}{0.48} & \multirow[t]{2}{*}{0.49} & 29.2 & \multirow[t]{2}{*}{0.23} & \multirow[t]{2}{*}{0.63} \\
\hline$>45$ & 25 & 31.7 & & & 36.1 & & \\
\hline \multicolumn{8}{|l|}{ Treatment date } \\
\hline Pre-2009 & 24 & 22.7 & \multirow[t]{2}{*}{2.32} & \multirow[t]{2}{*}{0.13} & 22.7 & \multirow[t]{2}{*}{6.13} & \multirow[t]{2}{*}{0.01} \\
\hline Post-2009 & 29 & 37.6 & & & 67.1 & & \\
\hline \multicolumn{8}{|l|}{ T stage } \\
\hline T3 & 10 & 62.5 & \multirow[t]{2}{*}{1.46} & \multirow{2}{*}{0.23} & 66.7 & \multirow[t]{2}{*}{1.36} & \multirow[t]{2}{*}{0.24} \\
\hline $\mathrm{T} 4$ & 43 & 18.4 & & & 23.8 & & \\
\hline \multicolumn{8}{|l|}{ N stage } \\
\hline N0-1 & 26 & 48.0 & \multirow[t]{2}{*}{7.42} & \multirow[t]{2}{*}{0.01} & 57.9 & \multirow[t]{2}{*}{7.46} & \multirow[t]{2}{*}{0.01} \\
\hline $\mathrm{N} 2-3$ & 27 & 8.0 & & & 9.4 & & \\
\hline \multicolumn{8}{|l|}{ Clinical stage } \\
\hline III & 9 & 80.0 & \multirow[t]{2}{*}{2.47} & \multirow[t]{2}{*}{0.12} & 80.0 & \multirow[t]{2}{*}{2.60} & \multirow[t]{2}{*}{0.11} \\
\hline IVa & 44 & 17.4 & & & 22.9 & & \\
\hline \multicolumn{8}{|l|}{ Pathological type } \\
\hline $\begin{array}{l}\text { Undifferentiated } \\
\text { non-keratinizing } \\
\text { carcinoma }\end{array}$ & 46 & 25.1 & 0.02 & 0.90 & 30.3 & \multirow[t]{2}{*}{0.15} & \multirow[t]{2}{*}{0.70} \\
\hline Other & 5 & 66.7 & & & 66.7 & & \\
\hline Radiotherapy meth & & & & & & & \\
\hline IMRT & 24 & 45.5 & 6.89 & 0.01 & 50.8 & 8.46 & 0.00 \\
\hline 2D-CRT & 29 & 9.1 & & & 12.7 & & \\
\hline
\end{tabular}

Survival rate was analyzed using Kaplan-Meier and log-rank analyses. T stage, tumor stage; N stage, stage of lymph node involvement; IMRT, intensity-modulated radiotherapy; 2D-CRT, two-dimensional conventional radiotherapy.

possessing stage IVb disease, based on the presence of stage N3 lymph node involvement, while the other 9 patients were classified as possessing stage III disease.

No significant difference in the OS rate of T3 and T4 patients with cervical spine involvement was identified in the present study using Cox multivariate regression analysis $\left(\chi^{2}=0.52 ; \mathrm{P}=0.47\right)$. The 5-year OS rate of all 53 patients was $25.5 \%$, and the 5 -year OS rate of the 24 patients treated by IMRT was $45.5 \%$. These rates were significantly decreased compared with the OS rate of patients with local advanced NPC (T3-T4) (2,3,11-15). Previous studies have also revealed that prevertebral space invasion was an independent prognostic factor in patients with NPC that were treated by IMRT $(4,16,17)$. Although it was rational to classify simple cervical spine involvement as T3 in precise radiotherapy, and IMRT increased the local control rate in patients with cervical spine involvement, the clinical treatment of NPC may become more challenging and the prognosis may be unfavorable if the prevertebral space or even cervical vertebrae was invaded by NPC $(12,13)$. Thus, additional studies are required to identify whether $\mathrm{T}$ stage should be adjusted in patients with cervical spine involvement.

The optimization of the radiation dose between the tolerance of the spinal cord and tumor targets is one of the most important issues in NPC patients with cervical spine involvement. Spinal cord radiation injuries are serious clinical complications that should be avoided in clinical radiotherapy, particularly severe grade III and IV radiation injury $(4,17)$. Since the tumor is extremely close to the affected spinal cord when cervical vertebrae are involved, it is extremely challenging to design clinical treatment protocols. In addition, radiation myelitis is often perplexing. When using 2D-CRT, the tumor target dose must be reduced due to the tolerance dose of the spinal cord (17). However, with IMRT the dose may be optimized based on the precision and degree of control over the radiation dose, which allows the tumor radiation dose to be increased significantly $(3,18-20)$. The maximum tolerance dose of the spinal cord in the IMRT group was $45 \mathrm{~Gy}$, compared to $40 \mathrm{~Gy}$ in the 2D-CRT group. A safety dose of 5 Gy was set to avoid scattering and treatment errors in 
Table IV. Cox multivariate prognostic analysis of clinical data.

\begin{tabular}{|c|c|c|c|c|c|c|c|c|c|c|c|c|}
\hline \multirow[b]{2}{*}{ Factors } & \multicolumn{4}{|c|}{ OS rate } & \multicolumn{4}{|c|}{ LPFS rate } & \multicolumn{4}{|c|}{ DMFS rate } \\
\hline & $\beta$ & $\mathrm{SE}$ & $\chi^{2}$ value & P-value & $\beta$ & SE & $\chi^{2}$ value & P-value & $\beta$ & SE & $\chi^{2}$ value & P-value \\
\hline $\begin{array}{l}\text { Radiotherapy } \\
\text { method }\end{array}$ & -1.52 & 0.54 & 8.02 & 0.01 & -2.59 & 0.71 & 13.10 & 0.00 & -1.10 & 0.66 & 2.84 & 0.09 \\
\hline Gender & 0.06 & 0.62 & 0.01 & 0.92 & 0.79 & 0.54 & 2.18 & 0.14 & -0.56 & 0.84 & 0.43 & 0.51 \\
\hline Age & -0.65 & 0.57 & 1.27 & 0.26 & -0.36 & 0.58 & 0.38 & 0.54 & -0.14 & 0.68 & 0.04 & 0.84 \\
\hline Treatment time & -0.69 & 0.71 & 0.93 & 0.33 & 1.07 & 0.60 & 3.20 & 0.07 & -0.14 & 0.86 & 0.03 & 0.87 \\
\hline T stage & 0.89 & 1.23 & 0.52 & 0.47 & 1.43 & 1.24 & 1.32 & 0.25 & -0.32 & 1.39 & 0.05 & 0.82 \\
\hline $\mathrm{N}$ stage & 0.89 & 0.30 & 8.53 & 0.00 & 1.01 & 0.36 & 8.16 & 0.00 & 0.84 & 0.39 & 4.59 & 0.03 \\
\hline Clinical stage & 0.10 & 1.75 & 0.00 & 0.95 & -2.30 & 1.53 & 2.24 & 0.13 & 1.04 & 1.97 & 0.28 & 0.60 \\
\hline Pathological type & 1.12 & 1.32 & 0.72 & 0.40 & -0.34 & 0.97 & 0.12 & 0.73 & -11.26 & 679.62 & 0.00 & 0.99 \\
\hline
\end{tabular}

OS, overall survival; LPFS, local progression-free survival; DMFS, distant metastasis-free survival; T stage, tumor stage; $\mathrm{N}$ stage, stage of lymph node involvement; SE, standard error.

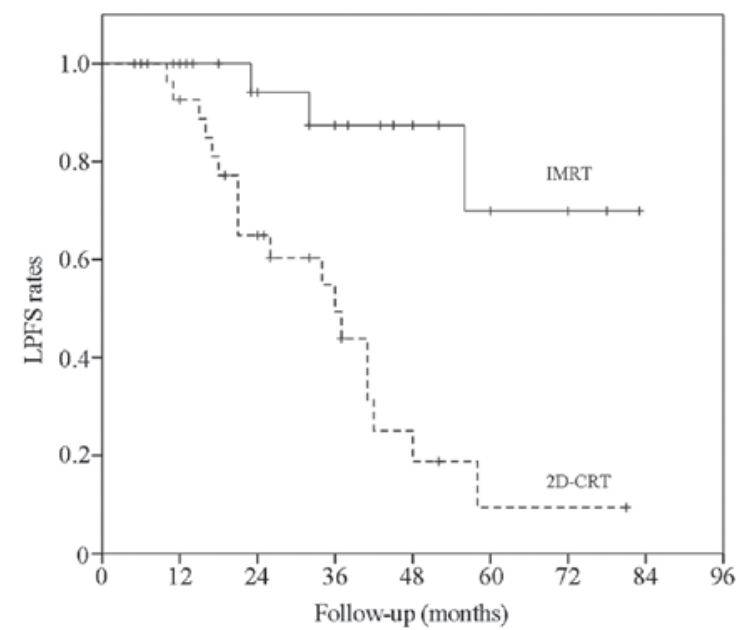

Figure 2. LPFS curves in the IMRT and 2D-CRT groups. The LPFS rates were assessed using Kaplan-Meier and Log-Rank analyses. The LPFS rates in the two groups were significantly different $\left[\chi^{2}=13.26 ; \mathrm{P}<0.01\right.$; IMRT 95\% confidence interval (CI), 59.71-82.94 months; 2D-CRT 95\% CI, 22.23-45.40 months]. IMRT, intensity-modulated radiotherapy; 2D-CRT, two-dimensional conventional radiotherapy; LPFS, local progression-free survival.

2D-CRT, due to the proximity of the posterior boundary of the radiation field, which was $5 \mathrm{~mm}$ from the vertebral canal, and the spinal cord. The lowest GTV dose of the involved cervical vertebrae was $60.3 \mathrm{~Gy}$ in the 24 patients in the IMRT group. However, the lowest GTV dose could not be higher than 45 Gy in the CRT group, as the radiation dose on the posterior region of the vertebral body could not be increased using the 2D anterior-posterior opposed field radiation that was adopted in the 2D-CRT group.

During 2D-CRT, the spinal cord must be avoided, so doses in regions of the tumor in the involved vertebral body were insufficient (21). There were three cases of radiation myelitis in the two groups, with an incidence of 5.88\% (3/53), which was significantly increased compared with the average incidence during each period of NPC (21-23). All 3 cases of radiation myelitis were degree I and occurred at 3-6 months subsequent

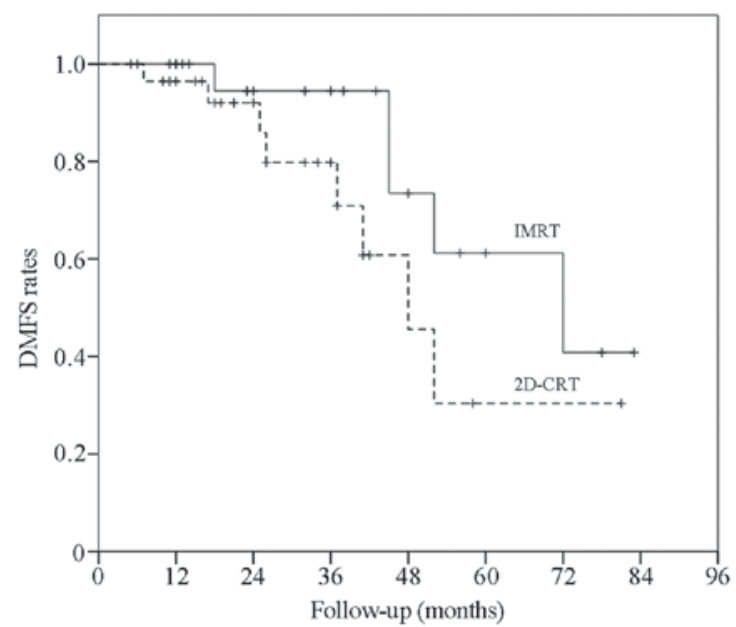

Figure 3. DMFS curves in the IMRT and 2D-CRT groups. The DMFS rates were assessed using the Kaplan-Meier and Log-Rank analyses. The DMFS rates in the two groups were significantly different $\left[\chi^{2}=2.29 ; \mathrm{P}=0.13\right.$; IMRT 95\% confidence interval (CI), 53.74-77.00 months; 2D-CRT 95\% CI, 38.27-64.47 months]. IMRT, intensity-modulated radiotherapy; 2D-CRT, two-dimensional conventional radiotherapy; DMFS, distant metastasis-free survival.

to radiotherapy. The symptoms were mild and mainly manifested as an electric shock sensation during head bowing and mild pain in the upper limbs. Out of the 3 cases, 1 occurred in the 2D-CRT group and 2 cases occurred in the IMRT group, 1 of which experienced nerve root compression symptoms prior to radiotherapy. Those symptoms were alleviated following radiotherapy, but the electric shock sensation during head bowing and mild pain in the upper limbs appeared 5 months subsequent to radiotherapy. The majority of the symptoms in the three patients were alleviated subsequent to treatment with hormones, neurotrophic drugs and vitamins, and radiation myelitis did not progress during the 2-year follow-up period.

The increased incidence of radiation myelitis in the IMRT group may be due to certain factors. As the cervical vertebrae were involved, broadening of the posterior boundary of the radiation field was attempted to increase the dose administered 
to the tumor target, leading to a relatively increased dose on the spinal cord, in particular in the IMRT group. Also, although the protocol was designed using the IMRT TPS and evaluated, errors were unavoidable during the clinical practice since the high dose region was usually $<5 \mathrm{~mm}$ from the spinal cord boundary, leading to uncertainty on the spinal cord dose. In addition, the IMRT dose was strongly controlled, the use of a large raditation dose was attempted to maximize the dose delivered to the spinal cord. By contrast, the dose of radiation administered to the spinal cord was unable to be controlled in the 2D-CRT group, leading to a more conservative administration of radiotherapy, and the incidence of radiation myelitis in the 2D-CRT group was decreased.

Although the occurrence of NPC with cervical spine involvement is rare, it is challenging to treat in clinical practice (5-7). In 2D-CRT, the radiation doses administered to the majority of tumors are inadequate due to the spinal cord tolerance to radiation, which results in a decreased control rate of the local tumor and affects patient prognosis and life quality. Prior to the introduction of IMRT, the majority of NPC patients with cervical spine involvement received palliative radiotherapy. The control rates of the local tumor were low in the majority of patients, and the 3- and 5-year survival rates of these patients were significantly decreased compared with the survival rates of patients without cervical spine involvement $(4,17)$.

Conformal IMRT was introduced as a treatment for NPC at The First Affiliated Hospital of Bengbu Medical College in 2006, and 53 patients with NPC that demonstrated cervical spine involvement have been treated with conformal IMRT since that time. Out of these 53 patients, 24 patients received IMRT, and the other 29 patients were administered with 2D-CRT, based on individual circumstances. The IMRT group demonstrated significantly improved 3- and 5-year OS and LPFS rates compared with the 2D-CRT group. However, no difference was observed between the 3- and 5-year DMFS rates of the two groups.

The differences between the clinical efficacy in the two therapies may be due certain factors. In 2D-CRT, this difference may be due to the exposure doses of the tumors that invaded into vertebral bodies being restricted by the spinal cord dose limit, and only tumors that are highly sensitive to radiation may be completely eliminated by 40 Gy radiotherapy. Local control and long-term survival may only be achieved in patients with sensitive tumors. 2D-CRT only acts as a palliative treatment for those patients with residual tumor tissue in vertebral bodies subsequent to $40 \mathrm{~Gy}$ radiotherapy (22). These differences may occur in IMRT, since the target region in the involved cervical vertebrae may be precisely defined. Therefore, the exposure doses of the tumor tissues invading the cervical vertebrae are much higher compared with the doses in 2D-CRT. Thus, local control is significantly improved in IMRT compared with 2D-CRT (19).

The results of the present study revealed that the low limit of the exposure dose of the target region in involved cervical vertebrae was $40 \mathrm{~Gy}$ in the 2D-CRT group, compared to $60.3 \mathrm{~Gy}$ in the IMRT group. The local complete response rate in the IMRT group was $75.0 \%$ (18/24). However, it was only $25.9 \%$ $(7 / 27)$ in the 2D-CRT group. There was no difference between the DMFS rates of the two groups, which suggested that distant
NPC metastasis was affected by numerous factors and may not be altered through the improvement of local radiotherapy.

The most important advantages of IMRT are the protection of normal tissues and the controllability of the doses to OAR surrounding the radiation field. The present study demonstrated that IMRT may provide improved dose limiting and protection to parotid glands compared with 2D-CRT, with significant difference in the incidence of dry mouth between the two groups $\left(\chi^{2}=7.11 ; \mathrm{P}=0.01\right)$. However, the incidence of myelosuppression $\left(\chi^{2}=0.58 ; \mathrm{P}=0.45\right)$ and oropharyngeal mucosal injury $\left(\chi^{2}=0.13 ; \mathrm{P}=0.72\right)$ was similar in the IMRT and 2D-CRT groups.

The 5-year survival rate of patients with NPC has increased to at least $70 \%$ with the use of IMRT, in contrast to $50 \%$ in patients treated with 2D-CRT. Even the 5-year survival rate of patients with advanced NPC may increase if they are treated properly $(18,19)$. Previous studies have revealed that IMRT is valuable in the clinical treatment of patients with NPC, in particular for locally advanced and recurrent NPC subsequent to radiotherapy $(20,24)$. As revealed in the present study, IMRT improves the local control and survival rate and also reduces radiation side-effects experienced by patients.

For NPC patients with cervical spine involvement, conformal IMRT has significant advantages over 2D-CRT in local control and survival. This type of patient should be treated with IMRT to gain maximal treatment benefits. However, with the option of precise radiotherapy, additional studies are required to explore whether cervical spine involvement remains an important prognostic factor for patients with NPC.

\section{Acknowledgements}

This study was supported by the Twelfth Five-Year Key Clinical Specialties Construction Fund of Anhui Province (grant no. 01Z33) and the Provincial Project of Natural Science Research of Anhui Province of China (grant no. 1408085MH190)

\section{References}

1. Liu Q, Chen JO, Huang QH and Li YH: Trends in the survival of patients with nasopharyngeal carcinoma between 1976 and 2005 in Sihui, China: A population-based study. Chin J Cancer 32: 325-333, 2013.

2. Sun Y, Tang LL, Chen L, Li WF, Mao YP, Liu LZ, Lin AH, Li L and Ma J: Promising treatment outcomes of intensity-modulated radiation therapy for nasopharyngeal carcinoma patients with NO disease according to the seventh edition of the AJCC staging system. BMC Cancer 12: 68, 2012.

3. Peng G, Wang T, Yang KY, Zhang S, Zhang T, Li Q, Han J and Wu G: A prospective, randomized study comparing outcomes and toxicities of intensity-modulated radiotherapy vs. conventional two-dimensional radiotherapy for the treatment of nasopharyngeal carcinoma. Radiother Oncol 104: 286-293, 2012.

4. Lee CC, Chu ST, Chou P, Lee CC and Chen LF: The prognostic influence of prevertebral space involvement in nasopharyngeal carcinoma. Clin Otolaryngol 33: 442-449, 2008.

5. Abdel Khalek Abdel Razek A and King A: MRI and CT of nasopharyngeal carcinoma. AJR Am J Roentgenol 198: 11-18, 2012.

6. Poon PY, Tsang VH and Munk PL: Tumour extent and T stage of nasopharyngeal carcinoma: A comparison of magnetic resonance imaging and computed tomographic findings. Can Assoc Radiol J 51: 287-295, 2000.

7. Zhang SX, Han PH, Zhang GQ, Wang RH, Ge YB, Ren ZG, Li JS and Fu WH: Comparison of SPECT/CT, MRI and CT in diagnosis of skull base bone invasion innasopharyngeal carcinoma. Biomed Mater Eng 24: 1117-1124, 2014. 
8. Edge S, Fritz AG, Byrd DR, Greene FL and Compton CC: Pharynx (including base of tongue, soft palate and uvula). In: AJCC Cancer Staging Manual. Edge S, Bryd DR, Compton CC, Fritz AG, Greene FL and Trotti A (eds). 7th edition. Springer, New York, NY, pp41-56, 2010.

9. World Medical Association: World Medical Association Declaration of Helsinki: Ethical principles for medical research involving human subjects. JAMA 310: 2191-2194, 2013.

10. Purdy JA: Current ICRU definitions of volumes: Limitations and future directions. Semin Radiat Oncol 14: 27-40, 2004.

11. Zong JF, Lin SJ, Zhang Z, Chen YB, Quo QJ and Pan JJ: Comparative study of nasopharyngeal carcinoma staging system between the Chinese 2008 and 1992 Fuzhou. Chin J Radiat Oncol 19: 481-485, 2010 .

12. Chen L, Liu LZ, Chen M, Li WF, Yin WJ, Lin AH, Sun Y, Li L and Ma J: Prognostic value of subclassification using MRI in the t4 classification nasopharyngeal carcinoma intensity-modulated radiotherapy treatment. Int J Radiat Oncol Biol Phys 84: 196-202, 2012.

13. Cao CN, Luo JW, Gao L, Yi JL, Huang XD, Wang K, Zhang SP, $\mathrm{Qu}$ Y, Li SY, Cai WM, et al: Clinical outcomes and patterns of failure after intensity-modulated radiotherapy for T4 nasopharyngeal carcinoma. Oral Oncol 49: 175-181, 2013.

14. Liu MT, Hsieh CY, Chang TH, Lin JP, Huang CC and Wang AY: Prognostic factors affecting the outcome of nasopharyngeal carcinoma. Jpn J Clin Oncol 33: 501-508, 2003.

15. Chen CY, Han F, Zhao C, Lu LX, Sun Y, Liu XF and Lu TX: Treatment results and late complications of 556 patients with locally advanced nasopharyngeal carcinoma treated with radiotherapy alone. Br J Radiol 82: 452-458, 2009.

16. Feng AC, Wu MC, Tsai SY, Chan KY, Cheng SH, Wang A, Chen SS, Jian JJ, Terng SD and Huang AT: Prevertebral muscle involvement in nasopharyngeal carcinoma. Int J Radiat Oncol Biol Phys 65: 1026-1035, 2006

17. Zhou GQ, Mao YP, Chen L, Li WF, Liu LZ, Sun Y, Chen Y, Tian L, Lin AH, Li L, et al: Prognostic value of prevertebral space involvement in nasopharyngeal carcinoma based on intensity-modulated radiotherapy. Int J Radiat Oncol Biol Phys 82: 1090-1097, 2012.
18. Leung SW and Lee TF: Treatment of nasopharyngeal carcinoma by tomotherapy: Five-year experience. Radiat Oncol 8: 107, 2013.

19. Lai SZ, Li WF, Chen L, Luo W, Chen YY, Liu LZ, Sun Y, Lin AH, Liu MZ and Ma J: How does intensity-modulated radiotherapy versus conventional two-dimensional radiotherapy influence the treatment results in nasopharyngeal carcinoma patients?. Int J Radiat Oncol Biol Phys 80: 661-668, 2011.

20. Han F, Zhao C, Huang SM, Lu LX, Huang Y, Deng XW, Mai WY, Teh BS, Butler EB and Lu TX: Long-term outcomes and prognostic factors of re-irradiation for locally recurrent nasopharyngeal carcinoma using intensity-modulated radiotherapy. Clin Oncol (R Coll Radiol) 24: 569-576, 2012.

21. Siala W, Mnejja W, Khabir A, Ben Mahfoudh K, Boudawara T, Ghorbel A, Frikha M and Daoud J: Late neurotoxicity after nasopharyngeal carcinoma treatment. Cancer Radiother 13: 709-714, 2009.

22. Lee AW, Law SC, Ng SH, Chan DK, Poon YF, Foo W, Tung SY, Cheung FK and Ho JH: Retrospective analysis of nasopharyngeal carcinoma treated during 1976-1985: Late complications following megavoltage irradiation. Br J Radiol 65: 918-928, 1992.

23. Teo PM, Leung SF, Chan AT, Leung TW, Choi PH, Kwan WH, Lee WY, Chau RM, Yu PK and Johnson PJ: Final report of a randomized trial on altered-fractionated radiotherapy in nasopharyngeal carcinoma prematurely terminated by significant increase in neurologic complications. Int J Radiat Oncol Biol Phys 48: 1311-1322, 2000.

24. Hua YJ, Han F, Lu LX, Mai HQ, Guo X, Hong MH, Lu TX and Zhao C: Long-term treatment outcome of recurrent nasopharyngeal carcinoma treated with salvage intensity modulated radiotherapy. Eur J Cancer 48: 3422-3428, 2012. 